\title{
A wireless system for sport assessment
}

\author{
Anna Lina Ruscelli *, Gabriele Cecchetti *, Mirco Manciulli ${ }^{\dagger}$, Piero Castoldi * \\ ${ }^{*}$ Scuola Superiore S. Anna, Pisa, Italy \\ Email: $\{$ a.ruscelli I g.cecchetti I p.castoldi $\} @$ santannapisa.it \\ ${ }^{\dagger}$ TIME Group, Lucca, Italy \\ Email: mirco.manciulli@timegroup.it
}

\begin{abstract}
In the context of sport assessment, the evaluation and monitoring of the referees decisions is of interest for several sports in order to avoid disputes and assist the referees in their activity. Some current solutions are based on video recording, third referee, etc. In this paper, a new wireless wearable system, result of the Italian research project REC-VISIO, is described suitable to assess the referees actions when they are on the move. The system is able to collect the referee staff visual perspective of a sport match, whose subjective is recorded, preprocessed and sent by a wireless network to a sideline workstation for final stabilization. The system architecture design and implementation are described along with the experimentation on the field, considering as use case a football match. The experimentation has shown the successful integration of all the different system components, where the cooperation of sensor-based subsystem, processing unit, and communication subsystem allows to collect and stabilize the video streaming reducing the effects of the movement of the referee, and send in real-time the video content to the sideline workstation without video quality degradation.
\end{abstract}

Keywords-Sport assessment, wireless system, embedded system, networking, smart assessment, smart social activities, smart computing.

\section{INTRODUCTION}

The pervasive diffusion of smart computing in the different sectors of the human life such as industry, agriculture, health, social activities, entertainment, sport improves their efficiency and the way people participate and opens new perspectives. In the context of sports activities, one of the topics where smart computing can impact is the sport assessment, intended as monitoring and evaluation of athletes performances. In general, the sport assessment deals with the evaluation, on a sport-specific basis, of the physical and mental conditions of athletes (strength, flexibility, power, endurance, posture, speed, balance, and agility, etc.), along with health conditions (cardiovascular health, pulmonary function, lactate threshold, neuromuscular strength, etc.). The aim is the evaluation of the athletes performance and health conditions before, during, and after the activity and the prevention of injuries such as cardiovascular risk or mild traumatic brain injury [1], [2].

Moreover, in the last years, the sport assessment is considering also further aspects such as the decision process of referees, whose actions heavily impact on the results of a sport competition. Indeed, referees are subject to the same physical conditions monitoring of the athletes, for instance by means of Electronic Performance and Tracking Systems (EPTS) [3], [4] (Optical-based tracking system, Local positioning system, GPS/GNSS satellite system), that monitor the athletes activity and can deal on video recording. However, previous considerations suggested to monitor also their actions introducing tool suitable to solve and avoid conflicts during a sport match. For instance, in the case of football, the Video Assistant Referees (VAR) based on the analysis of the video streaming by a team composed of a video assistant referee and three assistants, all top FIFA (International Federation of Association Football) match officials, is used to review the referee decisions who is notified of a possible wrong decision, can check the video on the involved action, confirm the decision or accept the suggested correction [5]-[7]. In particular, VAR is effective in the case of goals, penalty decisions, red card incidents, mistaken identity. However, this tool is simply based on video recording from different points of view on the sideline of the field of game and on their analysis by sport referees. No advanced technologies, nor further data with respect to the video streaming are used. For instance, the monitoring of referee point of view of the scene is missing that can help to understand the referee process decision. In this context, smart computing can provide advanced technological tools and equipment to monitor, control, and evaluate a wide set of parameters allowing a real-time collection and evaluation of data when the athletes are on the move, and the storage of the data for advanced analysis.

In this paper a new wireless wearable system for the support of the referee decisions is proposed. This system is the result of the activity of the Italian research project REC-VISIO (Detection and Recording System of the point of view of the referee staff), financed by the Italian Tuscany region under the POR- FERS 2014-2020 (Regional Operational Program of the European Regional Development Fund) program. In particular, the experimented system is based on a wearable equipment that has been patented [8] and is able to collect the referee staff visual perspective of a sport match, for instance a football match, whose subjective is recorded, pre-processed and sent by means of a wireless network to a sideline workstation for further elaboration in order to support the referee decision and provide a tool for its assessment.

The aim is introducing a tool suitable to assess the referee behavior, to help the training of new referees, also providing additional video contents that can be delivered to sport TV channels. In particular, its innovative contribution with respect the state of the art of the sport assessment tools, such as EPTS, or referee assessment tools such as VAR, is the use of the video of the referee visual perspective, whereas its 
further added value is the local video stabilization and the effective integration of off-the shelf components. The system is composed by a helmet and a belt where the different elements are placed (the wearable subsystem), and by a sideline workstation. In particular, in the helmet a smart camera collects the subjective vision of the referee, an Inertial Measurement Unit (IMU), with the same orientation of the camera, provides the information required for the video stabilization (delta pose and angular speed of each frame with respect to the previous one), an elaboration unit pre-processes the video streaming encoding and synchronizing the frames with the IMU data, and a microphone collects the audio. Transmission, storage, and power units are placed on the belt, far from the referee head. The sideline workstation deals with the stabilization of the pre-processed data sent in real-time by the wearable subsystem, reducing the jitter effects due to the referee movement without video quality degradation. In particular, a software stabilization tool was used to reduce the video jitter [9] instead of mechanical stabilization due to dimensions and weight. In order to successfully perform these different functions, the system is based on a strict cooperation between the elaboration unit, the sensor-based subsystem, the software for the stabilization of the collected data and the transmission subsystem, with the aim of overcoming the challenges related to its design and implementation. Indeed, the implementation of such a system implies the respect of particular requirements about its wearability (reduced size and weight, no impact on the bearer movement, consideration of electromagnetic risk and perception due to the head positioning), along with project requirements related to the collection of the visual perspective of the referee that is on the move. Focusing on project requirements, since the movement of the referee affects the video quality, a stabilization local to the sideline workstation implies the picking and adaptation of the more tailored stabilization algorithms and the selection of the appropriate elaboration unit. From its side, the encoding of the video frame and their synchronization with the IMU data require a sufficient elaboration capacity embedded in the wearable subsystem. Moreover, the wireless connection, that positively impacts on the wearability of the system by the referee, requires a careful planning of the network in order to ensure a full coverage in the whole field of game and the sending of the video streams to the sideline workstation without degradation. Finally, the system has to be autonomous from the point of view of the power for all length of a sport match.

The presented embedded system has been tested on field considering as use case a football match. The experimentation has demonstrated the successful integration of all the different components with the overall final results of a good quality video streaming of the visual perspective of the referee generated at the end point of the system, the sideline workstation.

\section{RELATED WORKS}

In this section the existing tools for the referee activity assessment are illustrated at the best of our knowledge, along with a summary of the regulations related to the referee equipment.

According to the Rules of the Football Game of FIFA and FIGC (Italian Football Federation) Official Decisions the mandatory equipment is composed by whistle, watch, red and yellow cards, notebook (or other tool to record the tender facts). Furthermore, referees may use additional equipment to communicate with the other match officials (the assistants and the fourth man) such as electronic flags, earphones, etc., for instance the Vokkero referee communication set [10], EPTS or other equipment for performance monitoring, see Fig. 1. In particular, EPTS can be of three categories, [3], [4]: a)

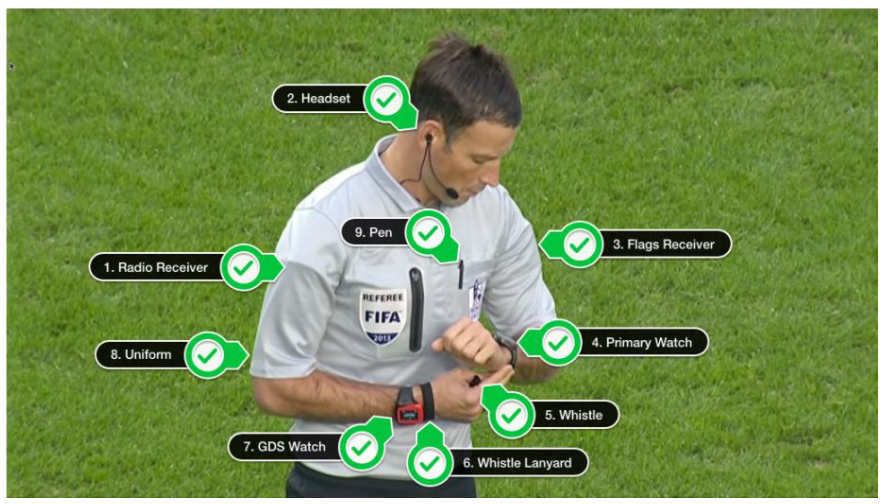

Figure 1. Standard referee equipment.

Optical-based tracking system, $b$ ) Local positioning system, $c$ ) GPS/GNSS satellite system. Optical-based tracking systems are the easiest solution commonly used in the football market, they are based on the video streams collected by cameras on stadium and are non-invasive to players, providing high sampling rate and possible ball tracking due to the use of high resolution cameras, without constraints about installation and communications. Drawbacks are the limited number of measurements due to the distance of cameras from the field and the manual corrections required in the case of tracking occlusions. In the case of Local positioning systems that are based on Local Positioning Unit worn by the players on their back, that sent data to a notepad in sideline, a high number of accurate real-time measurements is possible, whereas the use of Ultra Wide Band technology reduces the effects of interference in transmission path, with the drawbacks of fixed installation, and installation costs and time. Finally, in GPS/GNSS satellite systems, based on GPS unit worn on their back by the players, an high number of measurements is possible, short installation time is needed but the wearability and size of GPS unit and accuracy of measured data are an issue and it is required to ensure satellite signal line of sight in stadium. Furthermore, EPTS systems based on devices worn on the back of players markedly increase impact energy experienced at the upper-back during falls [11]. Only the first type of EPTS is able to collect video streams of the match but not related to the referee point of view directly from the field of match.

Focusing on the use of wearable cameras for referees, in Rugby, the more open to technologic innovations, during the Six 
Nations Championship the referees regularly wear cameras on the chest, and the use of the "Challenge" was introduced, where the video recordings are used during the game to facilitate the decisions of the referee. However, the positioning of the camera on the chest limits its use to more static game phases like scrums, where the alignment of the camera with the referee view is not affected by lateral movements of the head.

In basketball, both in USA and Europe, several experiments have been conducted with cameras worn by the referees, but through devices attached to the chest or embedded in the shirt, with the problems highlighted above, and where the live streaming of the video in subjective determines significant video jittering and very poor video quality. A camera worn on the head has been experimented without success being bulky and heavy for the wearer.

Finally, also in football (extremely conservative and reluctant to technological innovations) experiments were conducted with action cams worn by the referee, but they are heavy and with a strong visual impact. None of these experiments had a further technological developments, or systematic use. For instance, the Firstv1sion shirt with an embedded camera has been promoted in 2015 for basket and football, without any further evolution [12]. Furthermore, this equipment is without audio reception.

\section{THE WIRELESS WEARABLE SYSTEM FOR THE ASSESSMENT OF THE SPORT REFEREE DECISIONS}

In this section the wearable system for the assessment of the referee decisions based on the referee visual perspective of a sport match is described. In particular, the referee video subjective is recorded, pre-processed, and sent to a sideline workstation for the stabilization in order to support and motivate the referee decision. First of all, the design phase is described, where the different system requirements are derived by the evaluation of the current available tools and by an initial experimentation based on off-the-shelf devices. Then, the equipment prototype is described, focusing on its overall architecture and, then, on each subsystem (sensor-based subsystem, elaboration unit, communication subsystem, and power subsystem), highlighting their features and specifying and describing the physical components.

\section{A. Design phase and system requirements}

In order to define the system requirements an on field experimentation of an off-the-shell device as an example (the ultra light action camera Panasonic HX-A1MD) was done during training games of youth football teams and regional league games of youth basketball teams. This experimentation, along with the investigation about the state of the art in Section II, highlighted some limitations of the existing systems about their not optimal wearability, excessive weight, overheating of the head due to the electronics and the transmission equipment, perception of the electromagnetic risk due to the proximity to the head of the transmitter, and limited power autonomy of the supplied battery. Considering the stabilization carried out, at this preliminary stage, with the YouTube tool, a long processing time has experienced since this tool performs a correction by straightening the axes of each individual frame generating black squares, that have to be removed by a further specific software. Furthermore, the quality of the stabilized output was not yet satisfactory, due to persistent jittering problems.

All these considerations generated the following requirements for the proposed system:

- positioning on the head, in order to follow the movements of the head (rotations, in particular), that was not possible with cameras on the chest;

- minimum weight;

- greatest possible stability also through attachment to garment or to the body, without discomfort to the bearer;

- optic placed in axis and in a central position with respect to the eyes, in order to reproduce the view of the bearer.

Furthermore, the following features turned out to be essential:

- full HD video mode (high resolution);

- local use of video streams, with subsequent upload;

- real-time transmission of the video stream at a dockstation on the sideline;

- battery autonomy of at least one hundred minutes;

- integrated microphone.

\section{B. The embedded system architecture}

The previous considerations suggested to focus on a system that, aiming to collect video streams directly from the field of match, will have the following characteristics: wearable, wireless, and embedded. Indeed, being worn by the referee allows to collect the data related to the referee action directly on the source, in particular the physical configuration of the system will be suitable to collect the visual perspective of the referee. The system will be wireless in order to allow its wearability by the referee on the move, furthermore this makes the system more flexible and the installation easier. Due to the movement of the referee, the video is affected by the jitter and requires a stabilization in order to guarantee an adequate level of quality, thus the system will embed an elaboration unit to partially pre-process the data on-site. The functional system architecture will be composed by the following building blocks: sensors-based subsystem, elaboration unit, wireless transmission subsystem, storage and power unit. The components of the system, described in Fig. 2 are the following:

- a camera for the video recording,

- sensors for the derivation of the relative position of the frames,

- an elaboration unit for the local video processing,

- a transmission equipment to send the pre-processed data to the sideline workstation,

- a power unit to guarantee the energy autonomy.

Furthermore, the wearable system has been conceived as composed by a helmet to ensure the maximum stability of the component on the head considering the rapid movements of the referee, and a belt, where the different elements are deployed, as described in Fig. 2. In particular, in the helmet, whose first ergonomic study with the housing for elaboration unit and 


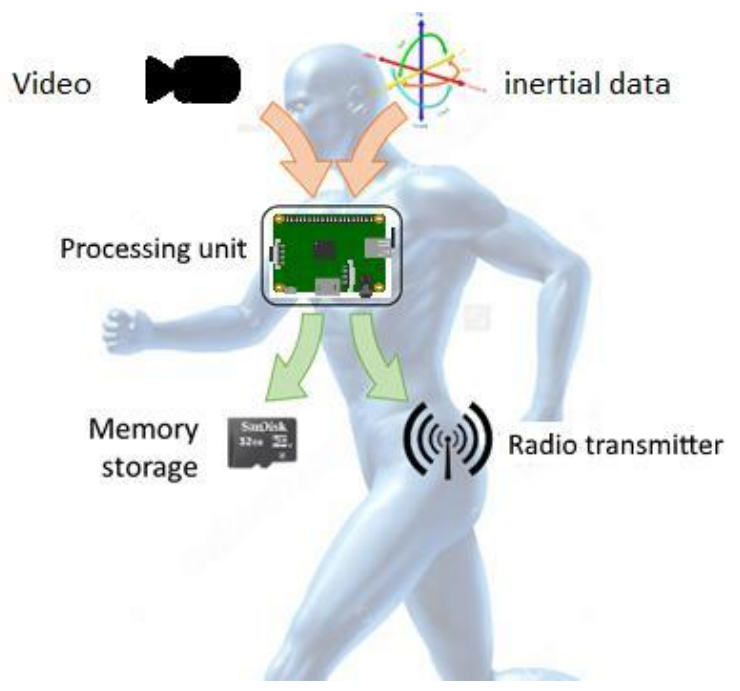

Figure 2. The wearable system architecture

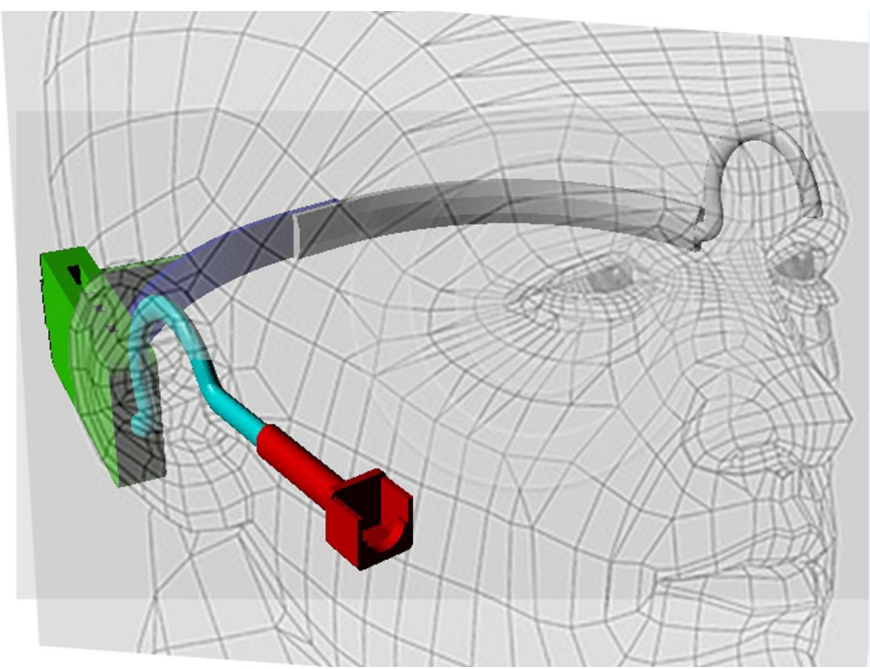

Figure 3. The ergonomic design of the wearable system on the head.

camera is illustrated in Fig 3, are placed the elaboration unit that pre-processes the video streaming, a smart camera that collects the subjective vision of the referee, and an Inertial Measurement Unit (IMU), based on accelerometers and gyroscopes, that sets the relative position of the frames. Instead, the transmission module and the power unit are placed on the belt in order to avoid the mentioned overheating and reduce the electromagnetic risk and its perception. In order to collect the data, compress and pre-process the video for the stabilization, and send in real-time the data to the sideline workstation without video quality degradation, the system is based on a strict cooperation between the elaboration unit, the sensor-based subsystem, the software platform for the elaboration of the collected data, and the transmission subsystem. In particular, the problem related to the live streaming of the video in subjective, which determines significant video jittering problems and very poor video quality, has been reduced by means of advanced video stabilization techniques and accurate design of the communication system.

The proposed system can be considered as solution alterna-

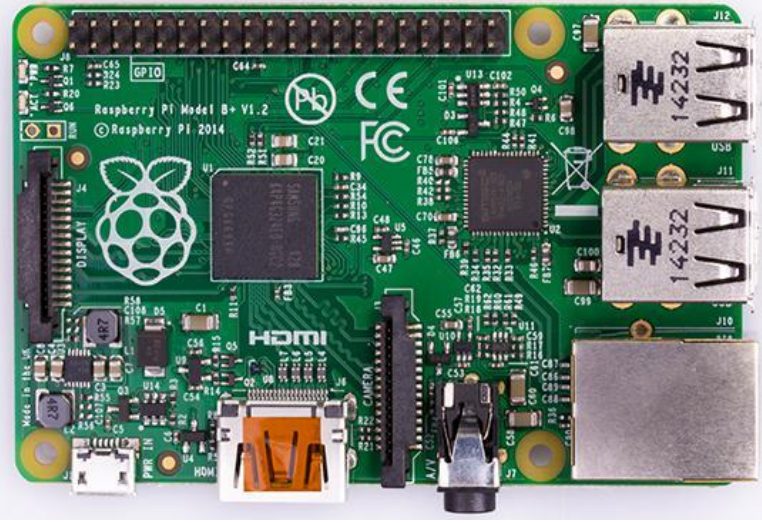

Figure 4. Raspberry Pi 1 Model B+ V1.2

tive to the currently available EPTS system based on wearable devices and it is apt also to monitor the athletes performances by the use of appropriate sensors.

1) The processing unit: For the local elaboration of video streams and signals from motion sensor the Raspberry $\mathrm{Pi}$ 1 Model B+ V1.2 (Fig. 4), a tiny computer for embedded application, was chosen due to its very low electrical absorption and very low overheating, so not heat dissipation systems are required. It features a Broadcom BCM2835 system on a chip, which includes a $700 \mathrm{MHz}$ ARM1176JZF-S processor, VideoCore IV Graphics Processing Unit (GPU), and RAM. It has $16 \mathrm{~KB}$ Level 1 cache and $128 \mathrm{~KB}$ Level 2 cache, that is used primarily by the GPU. Lower level output is provided by GPIO pins supporting common protocols like $\mathrm{I}^{2} \mathrm{C}$. The $\mathrm{B}+$ models have an Ethernet port and four USB ports. The O.S. is Raspbian, a Debian-based computer operating system.

The elaboration unit will compress the video streams following the H.264 standard, reducing the amount of information to send to the sideline station and will synchronize the frames with the IMU data.

2) The sensor-based equipment: The sensor-based equipment is composed by the camera and an inertial measurement unit suitable to determine the position of the head of the referee required for the stabilization. Consequently, both these components are bounded together in order to have an alignment between camera reference frame and IMU reference frame.

The Raspberry camera v1.3 was chosen as miniaturized optical component, see Fig. 5. This camera has HD lens with a resolution of $2592 \times 1944$ pixels, approximately 25 x $24 \mathrm{~mm}$ in size, equipped with microphone for audio recording and connected to the electronic card through a cable, whose length must be within $40 \mathrm{~cm}$ to avoid dispersion. The communication is based on a 15-pin MIPI Camera Serial Interface.

The IMU is the MPU-9150 (Fig. 6), a 9-axis MotionTracking device targeting low power, low cost, and high-performance requirements of consumer electronics equipment. It incorporates run-time calibration firmware. It combines two chips: the MPU6050, with a 3-axis gyroscope, a 3-axis accelerometer, and an onboard Digital Motion Processor capable of processing complex MotionFusion algorithms; and the AK8975, a 3-axis digital compass. The MPU-9150 communicates with the system 

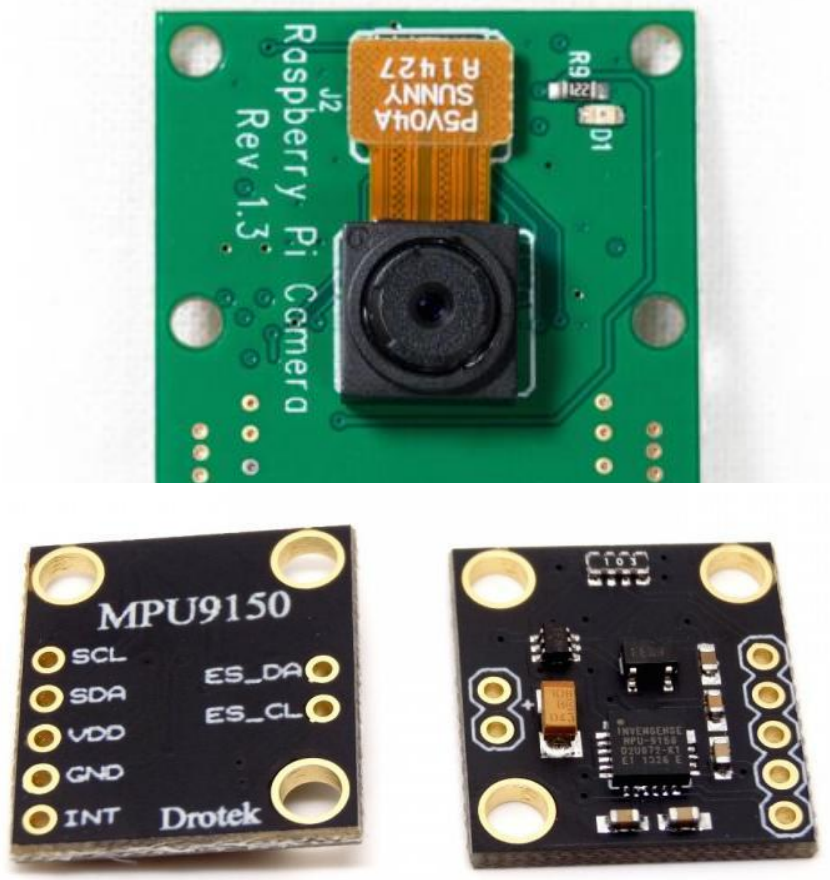

Figure 6. MPU-9150 IMU

processor using an $I^{2} C$ serial interface acting as a slave.

3) The communication subsystem: As explained above, the transmission devices are spread between the wearable equipment at the belt and the "ground" equipment to the field edge. This is the more critical subsystem being subject to the more difficult conditions that affect the performance of the application. In particular, the selection of the more suitable technology has to fulfill the trade-off between the following limitations and constraints:

- Transmission frequency - Network density: football, basketball, and rugby fields are congested from the point of view of wireless transmissions due to the audience presence. Thus, it is required to select frequencies not used by the on-field Access Points (AP) not part of the project.

- Transmission bandwidth: since the video resolution is 1920x1080 (Full-HD), a bandwidth between 2.5 and $10 \mathrm{Mbps}$ is required to ensure good performance, $20 \mathrm{Mbps}$ ensure optimal quality.

- Power source: the field equipment requires local power of $220 \mathrm{~V}$ available on the field board and transportable.

- Power consumption: since the referees wear the transmitter device a reduced power consumption is advisable.

- Unlicensed bandwidth: unlicensed Industrial, Scientific and Medical (ISM) bands reserved for non-commercial radio-communication applications.

- Type of transmission technology: off-the-shelf products, reduced cost, driver support.

- Wearable devices: the antennas for the communication with the field board have to be not invasive and robust.

- Transmission coverage of the AP: guarantee an adequate wireless connection when the referee moves along the whole extension of the field of game.

- Position and number of the Access Points: almost one AP, that receives all the data collected by the wearable equipment, is required for the communication between the referee and the field board.

- Reduced costs, driver support.

The evaluation of the available wireless technologies, such as Long Term Evolution (LTE), LTE Unlicensed (LTE-U), WiFi, radio frequencies (315 MHz and $433 \mathrm{MHz}$ ), suggested WiFi technology, based on the IEEE 802.11 standard [13]), as the most suitable for the wireless communications between the referee and the base station on the field of game sideline. Indeed, WiFi transmitters are available on the shelf, allowing the reduction of the equipment cost and, WiFi as consolidated technology guarantees the ease of finding COTS equipment with standard interfaces. IEEE 802.11 also operates on free frequency bands $(2.4$ and $5 \mathrm{GHz})$, which are part of the ISM band as desired, and it supports the point-to-point communication topology, that is required in this project. Thanks to these features, WiFi technology is frequently used for the transmission of multimedia traffic, [14]-[17]. IEEE 802.11 transmissions are robust to the interference thanks to the use of Spread Spectrum techniques. On the other hand, its diffusion implies bandwidth congestion affecting the quality of the transmission.

Considering the wearable devices, a USB WiFi dongle is directly connected to the Raspberry by means of a long USB cable and placed on the belt. Specifically the chosen Alpha Network AWUSO36AC complies 802.11ac, allowing to reach a $300 \mathrm{Mbps}$ data rate that fully satisfied the bandwidth needed for the video streaming, and the required coverage radius. The transmission sideline equipment will be described in the next section along with the description of the experimentation.

4) Power units: The components subject to overheating (WiFi dongle and power banks for feeding) are arranged on the belt, avoiding any problem due to overheating, and wearability of the device. The battery has an autonomy of two hours, sufficient for a football match and can be recharged through the micro USB port.

Fig. 7 describes the prototype of the helmet and Fig. 8 of the belt used for the on-field experimentation.

\section{ON-FIELD EXPERIMENTATION OF THE WIRELESS SYSTEM FOR THE SPORT ASSESSMENT}

In this section the on-field experimentation of the proposed system for the assessment of the referee decisions is described, focusing on the performances of the single components (elaboration unit for video stabilization and compression, and communication subsystem) and, then, considering the overall system. In particular, the evaluation of the wireless connectivity will be illustrated, describing the design of the overall wireless system composed by wearable equipment and wireless devices on the field for the transmission of the video stream from 


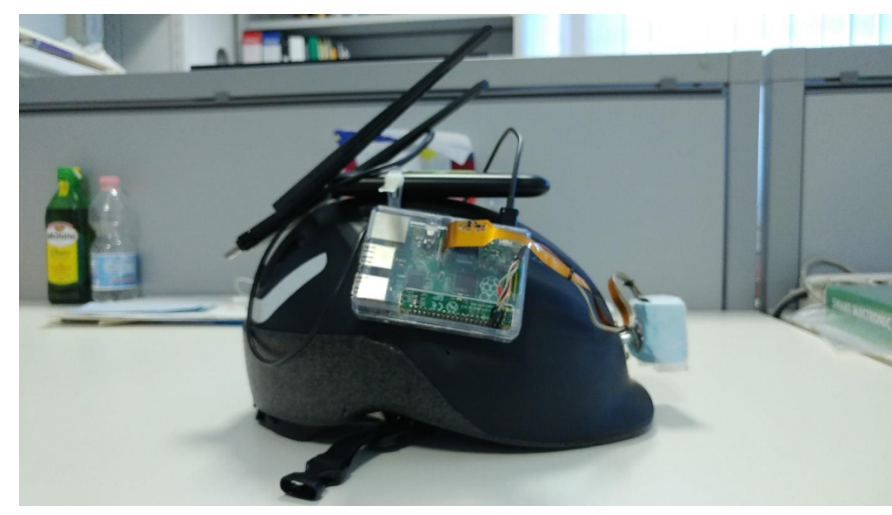

Figure 7. The prototype of the helmet.

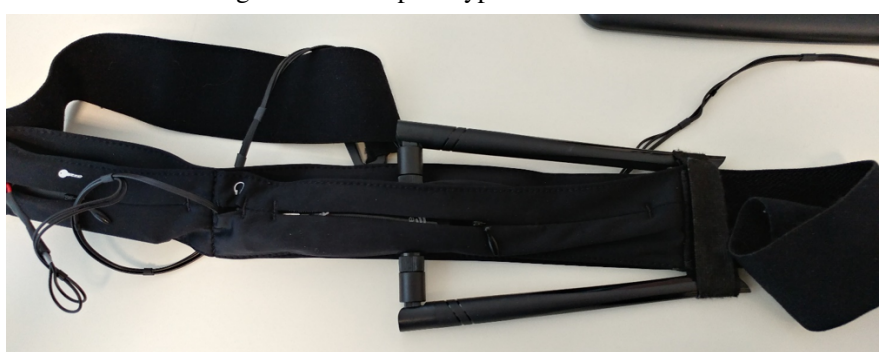

Figure 8. The prototype of the belt.

the referee to the sideline workstation, then the effectiveness of the stabilization software is illustrated and, finally, the onfield experimentation of the overall system will be presented, choosing a football math as use case.

\section{A. Wireless subsystem design and evaluation}

Focusing on the wireless communication between the referee and the sideline station, the main issue is to guarantee a sufficient level of signal throughout the field in order to dispatch the video stream to the workstation, independently from the position of the referee. Since the wearable equipment is not able to cover the overall field of game, some Access Points are required in order to forward the streams received from the referee to the board of the field with an adequate bandwidth. Consequently, the appropriate topology and distribution of APs and the selection of the most tailored off-the-shelf devices are required in order to guarantee that the referee wireless transmitter is always in their coverage area, taking into account the coverage radius of AP and wearable devices, the field of game dimensions. Furthermore, the wireless link is subject to the interference due other transmitting devices and due to the proximity to the field of game of the audience. The lowered position of the field of game with respect to audience stairs and their spatial separation, although minimal, minimally contribute to reduce the interference that remains relevant.

The dimensions and the coverage requirements of the football field of game are depicted in Fig. 9, where the maximum distance to be covered is equal to $108 \mathrm{~m}$.

The experimentation about the wireless coverage analyzed the performance of a wireless network with an increasing number of APs in order to determine their minimum number suitable to ensure the continuity of the signal without disconnections.

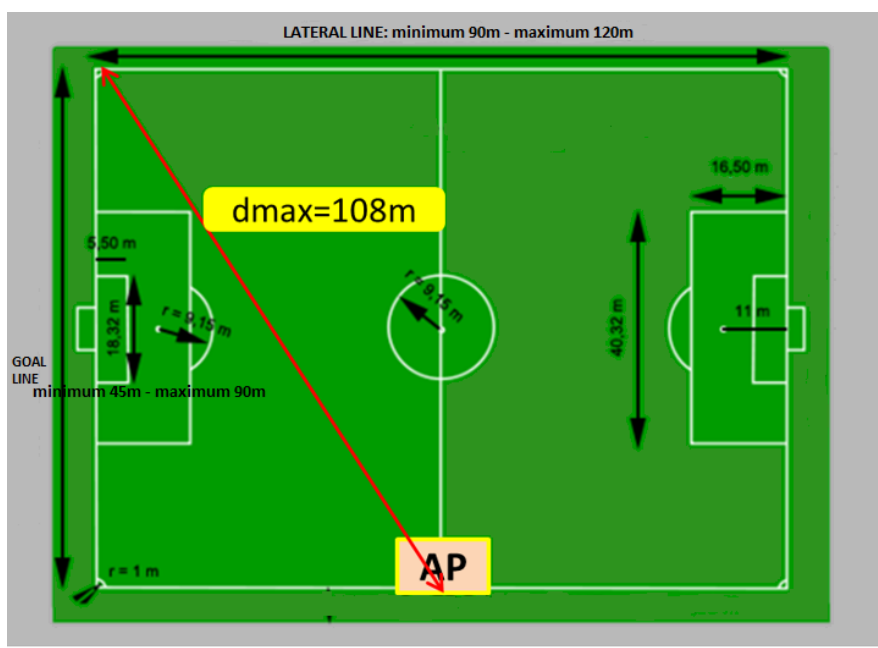

Figure 9. Worst case dimension of a football field of game (FIFA).

This analysis suggested that the optimal configuration was based on four APs deployed in the side of the field of game as show in Fig. 10, (where the ensured coverage is in green and the overlapping areas in violet), and using a mesh topology. This implies not only a connection between the wearable devices and the AP that covers the area where is the referee, but also a connection between the APs in order to deliver the data toward the AP nearest to the sideline base station. This allows to overcome the limitations due to the coverage radius of the single AP, avoiding too extensive coverage transmission that requires bigger antennas with different configurations. This topology is supported by a mesh WLAN (Wireless Local Area Network) that is composed by multiple nodes, the peers, that works as transmitter, receiver and repeaters to dispatch the data to the farthest nodes allowing covering longer distance than the coverage of a single node. The connection is based on the Fast Roaming, ruled by the IEEE 802.11r amendment of IEEE 802.11-2016, between the connected APs that provides a fast association to a new AP when the user is on the move. Furthermore, sectorial antennas are selected for the

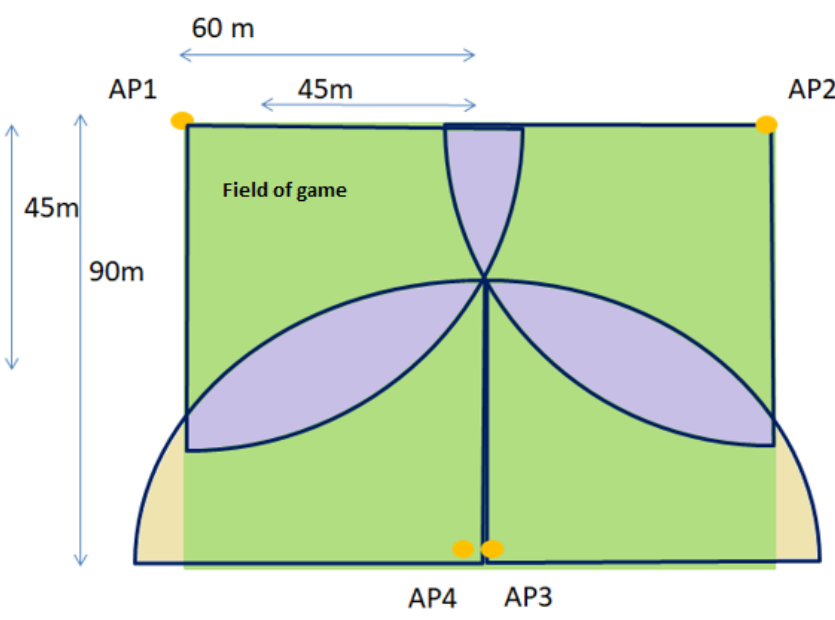

Figure 10. Distribution of APs with full coverage of the field of game. 
Table I

APs, whose sectorial radiation diagram is suitable to maximize the transmission power toward the direction to be covered, without illuminating unused areas such as that in the back of the antenna. The following equipment has been chosen for the test:

- n. 4 Ubiquiti Unifi Mesh AirMax UAP-AC-M,

- n. 2 Ubiquiti sectorial antennas MIMO 2x2 a $5 \mathrm{GHz}$ AM-5G17-90,

- n. 2 Ubiquiti sectorial antennas MIMO 2x2 a $5 \mathrm{GHz}$ AM-5G16-120,

- n. 8 low-loss cables to connect each of the four UAP-AC$\mathrm{M}$ to the corresponding antenna.

- n. 1 Ubiquiti switch US-8-150W.

Each UniFi AP-Outdoor dual band supports up to $300 \mathrm{Mbps}$ at $2.4 \mathrm{GHz}$ and $867 \mathrm{Mbps}$ at $5 \mathrm{GHz}$ with $2 \times 2 \mathrm{MIMO}$ technology and offers an outdoor range of up to $183 \mathrm{~m}$. A secondary Ethernet port can be used for bridging and for Power over Ethernet compliant to IEEE802.3af. Unifi UAP-AC-M supports Fast Roaming by default, that performs better than Zero handoff of previous generation devices. The client roaming is improved carefully acting on the minRSSI (Received Signal Strength Indicator) level of APs, make the regions partially overlapping, and then set a minRSSI level to force the client disassociation. Same SSID and key are used for each AP. The AP will be connected to a $2 \times 2$ MIMO dual polarity high gain sectorial antennas in substitution of the provided ones (two isotropic dual band antennas whose gain is $3 \mathrm{dBi}$ at 2.4 $\mathrm{GHz}$ and $4 \mathrm{dBi}$ at $5 \mathrm{GHz}$ ) in order to extend the coverage area: the gain is $16 \mathrm{dBi}$ for AM-5G16-120 with an opening angle of 120 degrees, and $17 \mathrm{dBi}$ for AM-5G17-90 with an opening angle of 90 degrees. The antennas work at $5 \mathrm{GHz}$, the less congested bandwidth. Finally, AirMax provide a proprietary Time Division Duplexing (TDD) access protocol of the mesh clients, with a traffic prioritization for video and voice streaming, and ruled by an intelligent AP controller, that, thanks to the ensured transmission time slot reduces the collisions problem.

As explained, only the $5 \mathrm{GHz}$ band was used in order to avoid the congestion issued related to the $2.4 \mathrm{GHz}$ band, that was disabled for all the devices. The Controller selected automatically channel 44 for all the devices, firstly for the APs and, then, for the associated clients.

In Table I the results about the test on the wireless coverage provided by the described deployment of the mesh network with the selected devices are illustrated. The physical rate and the consequent data rate were measured increasing the distance between referee and APs, considering links without disconnection. As shown, this configuration ensures the signal coverage for the maximum distance $(108 \mathrm{~m})$ and the required data rate. Thus, the set transmission subsystem ensures an easy support of the reduced number of referees, allowing the transmission of the data at the required rate.

\section{B. Stabilization subsystem evaluation}

The video streams affected by the jitter due to the referee movement was stabilized by a software components, based on
COVERAGE TEST RESULTS

\begin{tabular}{|l|l|l|}
\hline $\begin{array}{l}\text { Distance } \\
\text { (m) }\end{array}$ & $\begin{array}{l}\text { PHY rate } \\
\text { (Mbps) }\end{array}$ & $\begin{array}{l}\text { Data rate } \\
\text { (Mbps) }\end{array}$ \\
\hline 10 & 866 & $12-24$ \\
20 & 866 & $12-24$ \\
30 & 866 & $12-24$ \\
40 & 866 & $12-24$ \\
50 & 866 & $12-24$ \\
60 & 400 & $10-24$ \\
70 & 400 & $10-24$ \\
80 & 400 & $10-24$ \\
90 & 150 & $7-15$ \\
100 & 150 & $7-15$ \\
110 & 150 & $7-15$ \\
\hline
\end{tabular}

stabilization algorithms, [9]. Decoding and stabilization process takes place on the sideline base station. The process waits and collects video packets until a frame is ended, then it renders and stabilizes it, handling fragmentation of video packets. The upper-bound frame rate is 30fps (the video acquisition rate on Raspberry), that could decrease in the case of network congestion or any type of packet delay. In particular, Optical Flow [18] of the video (i.e. the distribution of the apparent velocities of objects in an image) and marker displacement through Object Tracking Technique was computed, using the Computer Vision Toolbox provided by Matlab to infer results. Optical Flow estimation is used in computer vision to characterize and quantify the motion of objects in a video stream, often for motion-based object detection and tracking systems. The Optical Flow constraint equation was solved using the Lucas-Kanade method [19] being the least sensitive to image noise, setting the Noise Threshold used for noise reduction. The evaluation of the stabilization algorithm was performed using a video with a black background where only a black cross marker on a white rectangle is visible. This gives the possibility to have the clearest possible results reducing image noise. Hence the camera was shaken causing the marker motions: in stable video the marker must move as little as possible, while in the not-stable video it can move freely. The effect of the stabilization was evaluated comparing the amount of motion in the stabilized video and in the not-stabilized one. As shown in Fig. 11, in the test the value of performance stabilization, i.e. the amount of motion in the stabilized video with respect to the not-stabilized one is equal to $46.82 \%$, meaning that almost the half of entire motions have been compensated.

\section{Experimentation of the wearable system}

After the experimentation of stabilization unit and wireless subsystem, the overall system was tested on field during a football match played in a regular field of game, using the described mesh topology, prototype, and video stabilization.

At the links https://www.dropbox.com/s/go5ocs6mtzqaw0u/ best_off_1_with_captions.mp4?dl=0 and https://www.dropbox. com/sh/tsqmkr4nggreiyt/AAAYGOI40V4U8UgxdehwLM3sa? $\mathrm{dl}=0$ the recording of the match with the comparison between the stabilized and the not-stabilized videos is shown. The experimentation has demonstrated the successful integration of all the different system components, where the cooperation of 


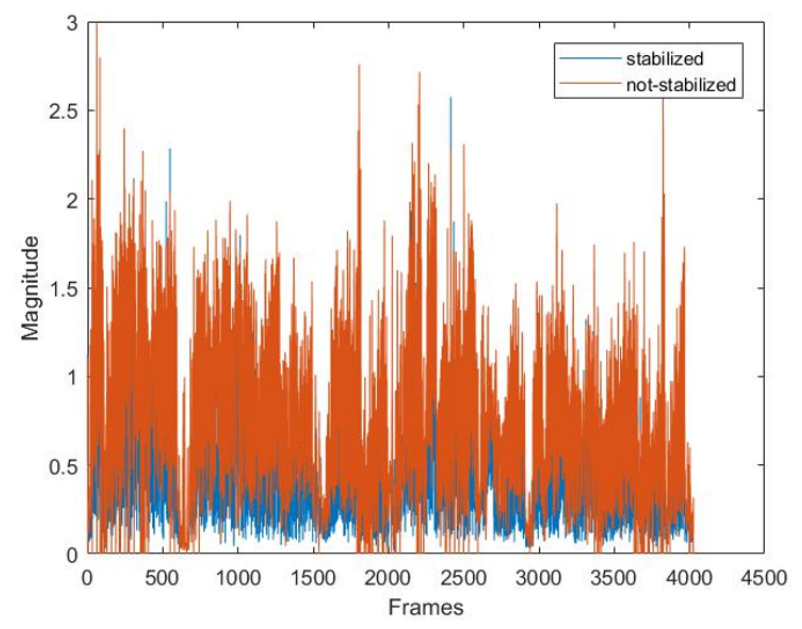

Figure 11. Performance of the video stabilization.

sensor-based subsystem, processing unit, and communication subsystem allows to collect and send in real-time the video content to the sideline workstation without meaningful quality degradation, and stabilize the video streaming reducing the effects of the movement of the referee.

This experimentation also has shown that the events of client roaming between the stations were infrequent, and often the client remained linked to an AP until the signal was very low or absent: such situation impacted on the received video quality for three times during the length of the match. Moreover, during the experimentation we switched off AP1 and AP2, slightly increasing the transmission power of AP3 and AP4. The system continued to work flawlessly, demonstrating that AP1 and AP2 were not essential to cover the movement of the referee.

\section{CONCLUSIONS AND FUTURE WORKS}

In this paper a wireless wearable system for the support of the referee decisions is proposed. This system is the result of the Italian research project REC-VISIO. The system is able to collect the referee staff visual perspective of a sport match, whose subjective is recorded, pre-processed and sent to a sideline field workstation for the stabilization.

The experimentation has demonstrated the successful integration of all the different system components, where the cooperation of sensor-based subsystem, processing unit, and communication subsystem allows to collect the video streaming, compress the data and synchronize the frames with the inertial data and send in real-time the video content to the sideline workstation without video quality degradation, where the software stabilization reduces the effects of the movement of the referee. The experimentations demonstrated the validity of the system, highlighting some possible improvements and future works. First of all, some changes in the ergonomic of the helmet could improve its wearability. Furthermore, the proposed system platform could be used also to monitor the athletes performances by the use of appropriate additional sensors.
Moreover, new fields of application were suggested. In particular, the system, opportunely modified, is currently under experimentation in the emergency ambulances. The aim is providing a tutoring tool for the ambulance personnel sending the video streaming of their subjective to the emergency control center, where a doctor can visualized the video in real-time, provide support for the assistance of blessed people and, consequently, organize the first aid service, route the ambulance to the tailored emergency center and alert that. Currently also this system is under patent.

\section{REFERENCES}

[1] S. Grindel, M. Lovell, and M. Collins, "The assessment of sportrelated concussion: The evidence behind neuropsychological testing and management," Clinical Journ. of Sport Medicine, vol. 11, no. 3, pp. 134-143, July 2011.

[2] C. Speers, A. N. Seth, K. C. Patel, D. J. Rakhit, and M. J. Gillett, "Defining the process of a cardiovascular risk assessment program: Lessons learned from cardiac assessment of elite soccer players in the United Kingdom," Clinical Journal of Sport Medicine, vol. 29, no. 6, pp. 500-505, 2019.

[3] FIFA, "Electronic performance and tracking systems," FIFA, Tech. Rep.

[4] D. Linke and D. M. Lames, "Validation of electronic performance and tracking systems EPTS under field conditions," PLOS ONE, vol. 13, no. 7, 2018.

[5] "VAR," https://football-technology.fifa.com/en/media-tiles/videoassistant-referee-var/.

[6] "Video assistant referee experiments," https://football-technology.fifa. com/en/media-tiles/video-assistant-referee-var-explained/.

[7] "University of Leuven research on VAR," https://www.leuvenmindgate. be/news/video-assistant-referee.

[8] M. Manciulli, P. Castoldi, G. Cecchetti, and A. Ruscelli, "Modello di utilita: Sistema di rilevamento indossabile interattivo," Italian Patent and Trademark Office (UIBM), Tech. Rep. 2020190000003344, 2019.

[9] A. Longobardi, F. Tecchia, M. Carrozzino, and M. Bergamasco, "A real-time video stream stabilization system using inertial sensor," in Proceedings of AVR, Santa Maria al Bagno, Italy, Jun 2019.

[10] "Vokkero," https://www.vokkero.com/en/35-referee-sets.

[11] M. Dunn, J. Hart, and D. James, "Wearing electronic performance and tracking system devices in association football: Potential injury scenarios and associated impact energies," in Proceeding of Biennial conference on the Engineering of Sport (ISEA), Brisbane, Australia, March 2018.

[12] "Firstvision camera," https://pt.slideshare.net/TelefonicaGrandesClientes/ ponencia-first-vision-startup-wayra-en-cloud-mediaday/6.

[13] IEEE802.11, "IEEE wireless LAN medium access control (MAC) and physical layer (PHY) specifications," IEEE, Piscataway, NJ, 2016.

[14] A. L. Ruscelli and G. Cecchetti, "A IEEE 802.11e HCCA scheduler with a reclaiming mechanism for multimedia applications," Advances in Multimedia, vol. 2014, doi:10.1155/2014/372693, no. ID 372693, 2014

[15] — " "Improving the QoS of IEEE 802.11e networks through imprecise computation," Int. Journal of Ad Hoc and Ubiquitous Computing, vol. 23, no. 3-4, pp. $152-167,2016$.

[16] A. L. Ruscelli, G. Cecchetti, and P. Castoldi, "Elastic QoS scheduling with step-by-step propagation in IEEE 802.11e networks with multimedia traffic," Wirel. Comm. and Mob. Comp., vol. 6, pp. 1-23, April 2019.

[17] A. L. Ruscelli, G. Cecchetti, A. Mastropaolo, and G. Lipari, "A greedy reclaiming scheduler for IEEE 802.11e HCCA networks," in Proceedings of ACM MSWiM, Miami Beach, Florida, nov 2011.

[18] S. Lim and A. El Gamal, "Optical flow estimation using high frame rate sequences," in IEEE Int. Conf. on Image Proc., 2001, pp. 925-928.

[19] D. Patel and S. Upadhyay, "Optical Flow measurement using Lucas Kanade method," Int. Jourm. of Comp. App., vol. 61, no. 10, Jan 2013. 